\title{
Electron-Tunneling Modulation in Percolating Network of Graphene Quantum Dots: Fabrication, Phenomenological Understanding, and Humidity/Pressure Sensing Applications
}

T. S. Sreeprasad, Alfredo Alexander Rodriguez, Jonathan Colston, Augustus Graham, Evgeniy Shishkin, Vasanta Pallem, and Vikas Berry*

Department of Chemical Engineering, Kansas State University, 1005 Durland Hall, Manhattan, Kansas, 66506, United States

Supporting Information

ABSTRACT: The two-dimensional (2D) electron cloud, flexible carbon-carbon bonds, chemical modifiability, and sizedependent quantum-confinement and capacitance makes graphene nanostructures (GN) a widely tunable material for electronics. Here we report the oxidation-led edge-roughening and cleavage of long graphene nanoribbons (GNRs) $(150 \mathrm{~nm}$ wide) synthesized via nanotomy (nanoscale cutting) of graphite (with $2 \mathrm{~nm}$ edged diamond knife) to produce graphene quantum dots (GQD). These GQDs $(\sim 100-200 \mathrm{~nm})$ selectively interfaced with polyelectrolyte microfiber (diameter $=2-20$ $\mu \mathrm{m})$ form an electrically percolating-network exhibiting a

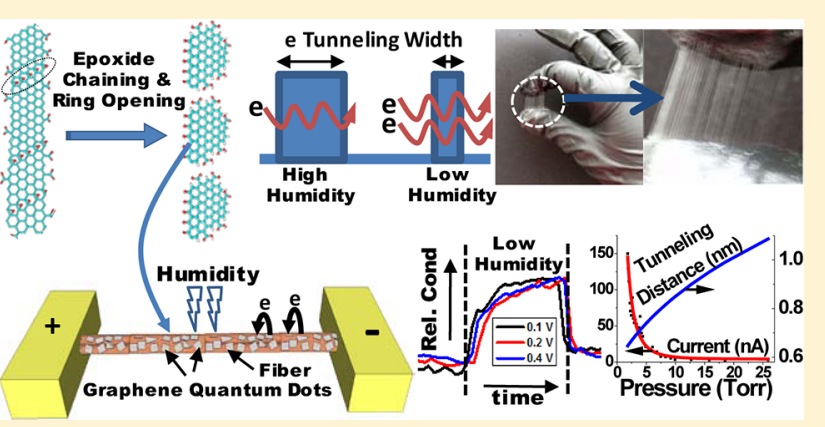
characteristic Coulomb blockade signature with a dry tunneling distance of $0.58 \mathrm{~nm}$ and conduction activation energy of $3 \mathrm{meV}$. We implement this construct to demonstrate the functioning of humidity and pressure sensors and outline their governing model. Here, a $0.36 \mathrm{~nm}$ decrease in the average tunneling-barrierwidth between GQDs (tunneling barrier $=5.11 \mathrm{eV}$ ) increases the conductivity of the device by 43-fold. These devices leverage the modulation in electron tunneling distances caused by pressure and humidity induced water transport across the hygroscopic polymer microfiber (Henry's constant $=0.215 \mathrm{Torr}^{-1}$ ). This is the foremost example of GQD-based electronic sensors. We envision that this polymer-interfaced GQD percolating network will evolve a new class of sensors leveraging the low mass, low capacitance, high conductivity, and high sensitivity of GQD and the interfacial or dielectric properties of the polymer fiber.

KEYWORDS: Graphene, quantum dots, electron tunneling, humidity sensor, nano arrays, microfibers

$G$ raphene quantum dots (GQDs) are atomically thick, conductive nanosheets of $\mathrm{sp}^{2}$ hybridized carbons, which exhibit size-, shape-, and edge-dependent electrical properties. ${ }^{1}$ The GQD structure is fundamentally different from other nanostructures, since its thickness reaches the "ultimate limit of dimensions" (single atom). ${ }^{2}$ The $\pi$-cloud in these single-atomthick GQDs provides delocalized charge carriers, imparting them with high conductivity. However, their electronic structure is governed by the edge construct (edge electronic states) and size (quantum confinement). ${ }^{3-5}$ Therefore, manipulating the size and edge characteristics of GQDs is critical for controlling their properties while the functionalization (similar to that of graphene) ${ }^{6}$ can alter their energy states.

While several processes have been reported to synthesize graphene quantum dots, ${ }^{1,7,8}$ the current challenges to be addressed to incorporate GQDs into applications include: (a) facile production of GQDs of controlled dimensions greater than $75 \mathrm{~nm}$, (b) routes for directed and selective assembly of GQDs into varied nanoarchitectures (for example percolating networks), and (c) interfacing GQDs with other functional materials for applications in electronic devices.
Self-assembly of nanomaterials has led to the realization of a wide range of phenomena, systems, and devices via complex structural design achieved by integration of nanostructures with conventional microelectronic circuitry. ${ }^{9-13}$ Such self-assembly has been applied in a wide range of devices, including solar cells, ${ }^{14}$ touch sensitive devices, ${ }^{15}$ and sensors. ${ }^{11}$ We present a novel synthesis process for GQDs from graphene nanoribbons (GNRs) and their facile self-assembly onto polymer microfibers to form a percolating GQD-network, which leverages the polymer's hygroscopic nature for applications as humidity and pressure sensors operating via electron-tunneling modulation. While several graphene-based-sensors exist, ${ }^{16-18}$ here we show a novel GQD based sensor. Moreover, it is important to note that most graphenic humidity-sensors are based on wateradsorption induced doping, which modulates the device conductivity. However, such sensors require high humidity levels (40-90\%) to achieve 1-9 fold conductivity modulation. ${ }^{19,20}$ Also, these sensors exhibit a decrease in conductivity

Received: January 27, 2013

Revised: $\quad$ March 13, 2013

Published: March 18, 2013 

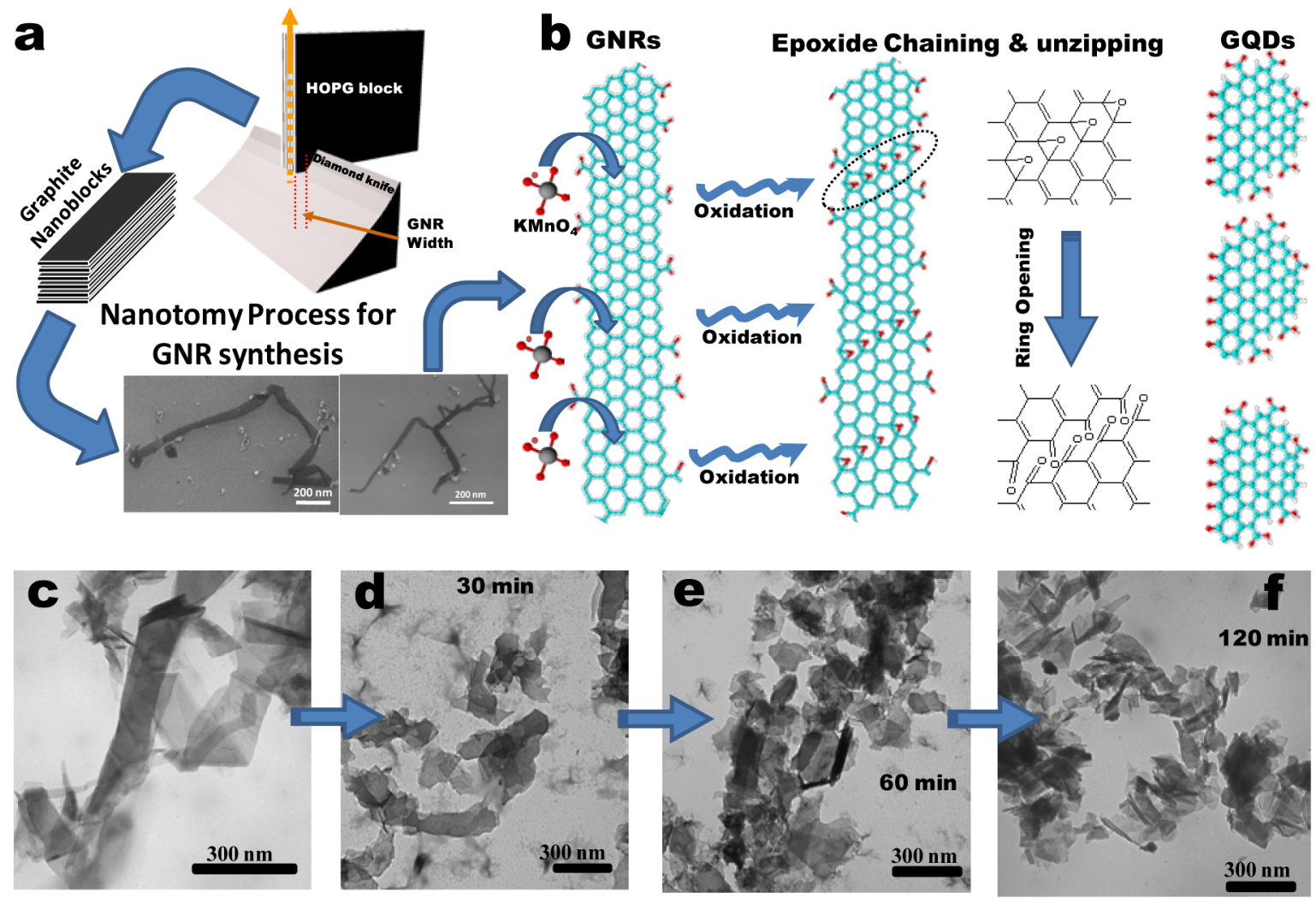

Figure 1. (a) Schematic illustrating the nanotomy process employed to synthesize GNRs, where a diamond knife ( 2 nm edge diameter) cleaves graphite into graphite nanoblocks, which are exfoliated to produce GNRs via mild oxidation. Representative FESEM micrographs of the exfoliated GNRs are shown in the bottom insets and panel c. (b) The reaction progression scheme shows the underlying mechanism depicting the conversion of GNRs to GQDs. (c-f) Transmission electron microscopy (TEM) images representing the chronological edge-roughening of GNRs and their cleavage during the conversion of GNRs into GQDs after 0 min (c, exfoliated GNRs), 30 min (d), 60 min (e), and 120 min (f) of GNR oxidation. The evolution of the structure of GQDs can be clearly observed. TEM images with isolated GQDs are shown in the Supporting Information.

upon removal of water from the graphene device. In the present work, (a) the GQD-network device exhibits an order of magnitude higher current modulation (43-fold) than any doping based graphene sensor (to the best of our knowledge); (b) the device operates in a lower humidity range: $0-40 \% \mathrm{rH}$ $(<0.007 \mathrm{~kg} / \mathrm{kg})$ and conduction increases with reduction in humidity; (c) a novel edge-roughening and cleavage process has been presented to convert GNRs into GQDs; and (d) to the best of our knowledge, this is the first GQD based electronic sensor.

In the first part of this work, the conversion of GNRs into GQDs via oxidative edge-roughening and cleavage are discussed (Figure 1). The GNRs were prepared via a nanotomy-based process ${ }^{1}$ that we reported in 2012. Briefly, graphite nanoblocks (GNBs), ultrathin sections of graphite, were cleaved using a diamond knife ( $2 \mathrm{~nm}$ sharp) to obtain the desired thickness. Each GNB consists of oriented stacks of GNRs $0.4 \mathrm{~nm}$ apart. A fixed weight of these GNBs were exfoliated via modified Hummer's method ${ }^{21}$ in a mild acidic environment, which oxidizes the GNR edges. These mildly oxidized GNRs were allowed to undergo a second round of Hummer's process, where the preoxidized GNRs were added into concentrated $\mathrm{H}_{2} \mathrm{SO}_{4}(1 \mathrm{~mL})$ kept in an ice bath and stirred. $\mathrm{KMnO}_{4}$ with a weight ratio of $1: 4\left(\mathrm{GNR} / \mathrm{KMnO}_{4}\right)$ was added slowly to avoid sudden rise in temperature. Four aliquots of the resultant sample were kept at $45{ }^{\circ} \mathrm{C}$ for different time intervals. This was followed by slow addition of $7 \mathrm{~mL}$ of distilled water to avoid temperature rise and then the addition of a few drops of $30 \% \mathrm{H}_{2} \mathrm{O}_{2}$ to arrest the reaction. The resultant solution was centrifuged and washed with $10 \% \mathrm{HCl}$ and distilled water several times, followed by redispersion in 8 $\mathrm{mL}$ of distilled water and $30 \mathrm{~min}$ of sonication. The samples subsequently underwent dialysis for 5 days to remove residual ions and contaminants. Finally, the solution was transferred to a glass vessel, sonicated again for $5 \mathrm{~min}$, and stored at room temperature $\left(25 \pm 2{ }^{\circ} \mathrm{C}\right)$. For the present study, $150 \mathrm{~nm}$ wide GNRs were used.

Figure $1 \mathrm{c}-\mathrm{f}$ shows the structural evolution of GNRs at different stages of the reaction as observed via transmission electron microscopy (TEM). These images show the edge roughening, cleavage, and size reduction process. The corresponding higher magnification micrographs with individual nanostructures are provided in the Supporting Information, Figure S2. Mildly oxidized GNRs (Figure 1a and c) were several micrometers long and $\sim 150 \mathrm{~nm}$ wide. Subsequent to Hummer's oxidation under highly acidic conditions at elevated temperatures for $30 \mathrm{~min}$ (Figure 1d): (a) most of the GNRs shorten in length varying between 500 and $700 \mathrm{~nm}$; (b) their edges become rougher; and (c) smaller nanostructures evolve. As the reaction proceeds, the length of the GNRs decreases progressively (Figure 1D-F) and edges roughen, and after 120 min of reaction most of the GNRs are converted to GQDs with smaller lateral dimensions (Figure 1f). Some rectangular quantum dots having a length around $200 \mathrm{~nm}$ were also observed ( $\sim 5 \%)$. After $240 \mathrm{~min}$ all of the GNRs were converted to GQDs, and no GNRs were observed (Supporting 

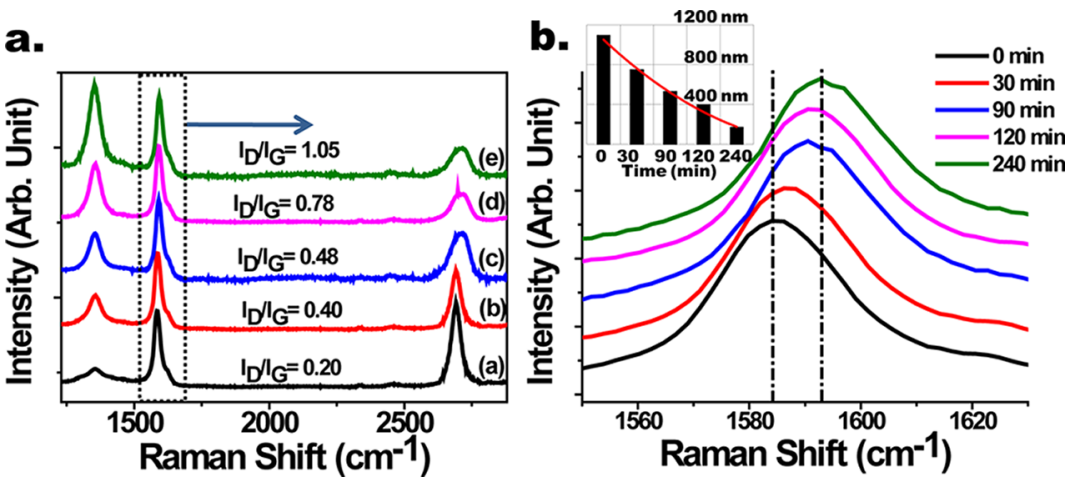

Figure 2. (a) Raman spectra of GQDs produced at different durations of oxidation of GNRs. The 2D peak dampens, and a prominent D-peak evolves with oxidation time. (b) Higher resolution view of the region marked in part a shows the shift in the G-peak position with reaction time. The inset shows the decrease in nominal diameter (obtained via DLS analysis) of GNRs with the evolution of reaction (more DLS results are presented in Supporting Information).
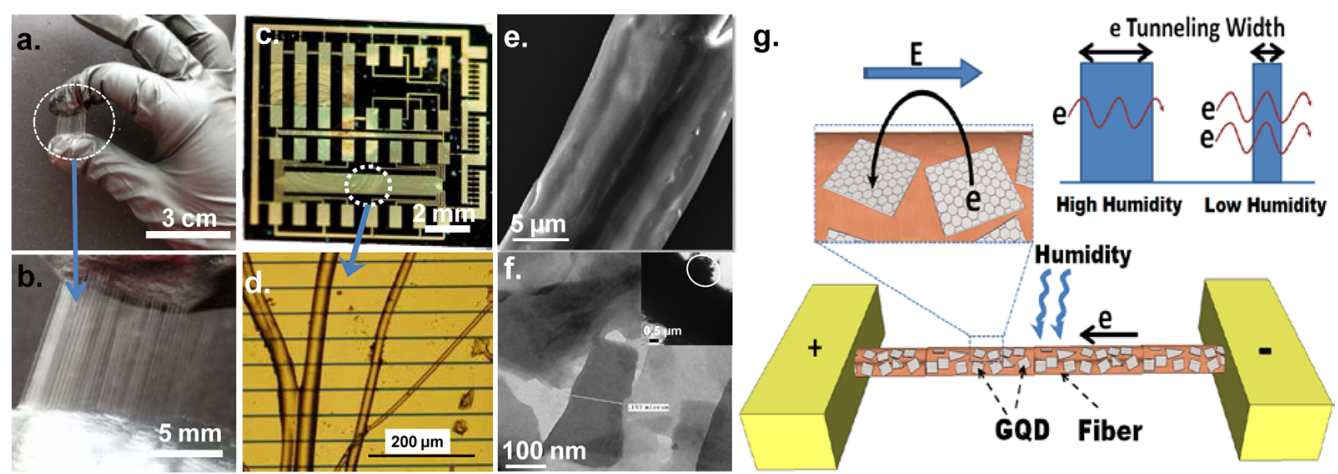

Figure 3. ( $\mathrm{a}$ and $\mathrm{b}$ ) Photographs depicting the process of direct spinning of long PAH microfibers from $40 \%$ PAH (aqueous) solution. These microfibers are then deposited on silica-substrate with gold electrodes. (c) Photograph of the silica chip shows the gold electrodes on silica-on-silicon wafer with PAH microfibers. This device construct was employed for humidity and pressure sensing. (d) Optical micrograph of the region outlined in part $c$ showing the microfibers bridging the electrodes. (e) An FESEM micrograph shows the GQDs forming a percolating network on PAHmicrofiber. (f) A high-magnification TEM micrograph of the GQDs immobilized on a PAH microfiber and forming a percolating network. The inset shows a low-magnification image of the PAH microfiber-GQD hybrid with the GQDs (marked with dotted circle and shown in f). (g) Schematic representation of the device construct and mechanism employed for characterization of electron-tunneling modulation in the GQD network and the humidity/pressure sensors.

Information, Figure S3). Representative TEM images of dispersed isolated GQDs are provided in the Supporting Information, Figure S4. The GQD size evolution was also studied by dynamic light scattering, which showed a systematic reduction in GQD size and disappearance of peak corresponding to the long axis of GNRs (inset of Figure $2 \mathrm{~b}$ and Supporting Information, Figure S5).

Raman spectroscopy was employed to analyze the evolution of structure of these GNs (Figure 2) (532 nm, 100× objective, $35 \mathrm{mV}$ with $10 \mathrm{~s}$ exposure). Here, samples after each phase of the reaction were drop-casted on a silica substrate and analyzed under a Raman spectroscope. Mildly oxidized GNRs showed two prominent features at $1585 \mathrm{~cm}^{-1}$ and $2694 \mathrm{~cm}^{-1}$ corresponding to $\mathrm{G}$ and $2 \mathrm{D}$ bands of graphene and a $\mathrm{D}$-peak at $1354 \mathrm{~cm}^{-1}$ representing the presence of edges and the isolated defects in the structure presumably due to the lowdegree oxidation. ${ }^{22}$ With the progression of the reaction, some general trends were observed: (a) the intensity of the $\mathrm{D}$ band progressively increases; (b) the $2 \mathrm{D}$ peak intensity progressively reduces; (c) the $2 \mathrm{D}$ peak broadens; (d) the $I_{\mathrm{D}} / I_{\mathrm{G}}$ ratio progressively increases; and (e) the position of $\mathrm{G}$ band shifts toward the higher wavenumbers. Further, the shift in the Gband was higher in the initial phase of the reaction and reduced as the reaction progressed. This suggests a reduction in the cleavage rate with progression of reaction as it reaches completion. In combination with TEM and DLS observations, the Raman spectra suggest that the exfoliated GNRs are only mildly oxidized. The $\mathrm{G}$ band shifting to higher wavenumber during oxidation indicates doping via oxidation, ${ }^{23}$ while the increase in the D-peak intensity implies (a) increase in the edge roughness, (b) increase in edge density due to the formation of more and smaller GQDs, and (c) conversion of the $\mathrm{sp}^{2}$ carbons into $\mathrm{sp}^{3}$ hybridized carbons via oxidation. ${ }^{22}$ These processes also reduce the intensity of $2 \mathrm{D}$ peak..$^{22,23}$ The increase in the intensity of the $\mathrm{D}$ band and attachment of oxygen functionalities are known to reduce the intensity of the 2D band and broaden it considerably. This observation is consistent with the oxidative cutting of GNRs and edge roughening. Further, we speculate that, since the gradient of the G peak position with laser power $\left(=0.143 \mathrm{~cm}^{-1} / \mathrm{mW}\right)$ is $\sim 58$ fold lower than that of supported graphene ${ }^{24}$ (see Supporting Information, Figure S6), the thermal conductivity of GQDs is at least an order of magnitude lower than graphene.

We believe that the conversion of GNRs to GQDs follows a mechanism similar to the oxidative cleavage $e^{25-28}$ of carbon nanotubes (CNTs) to short CNTs and of CNT unzipping into GNRs. ${ }^{8,29,30}$ Briefly, under acidic oxidation, epoxy groups cooperatively assemble in a line on the graphenic lattice, ${ }^{8}$ 

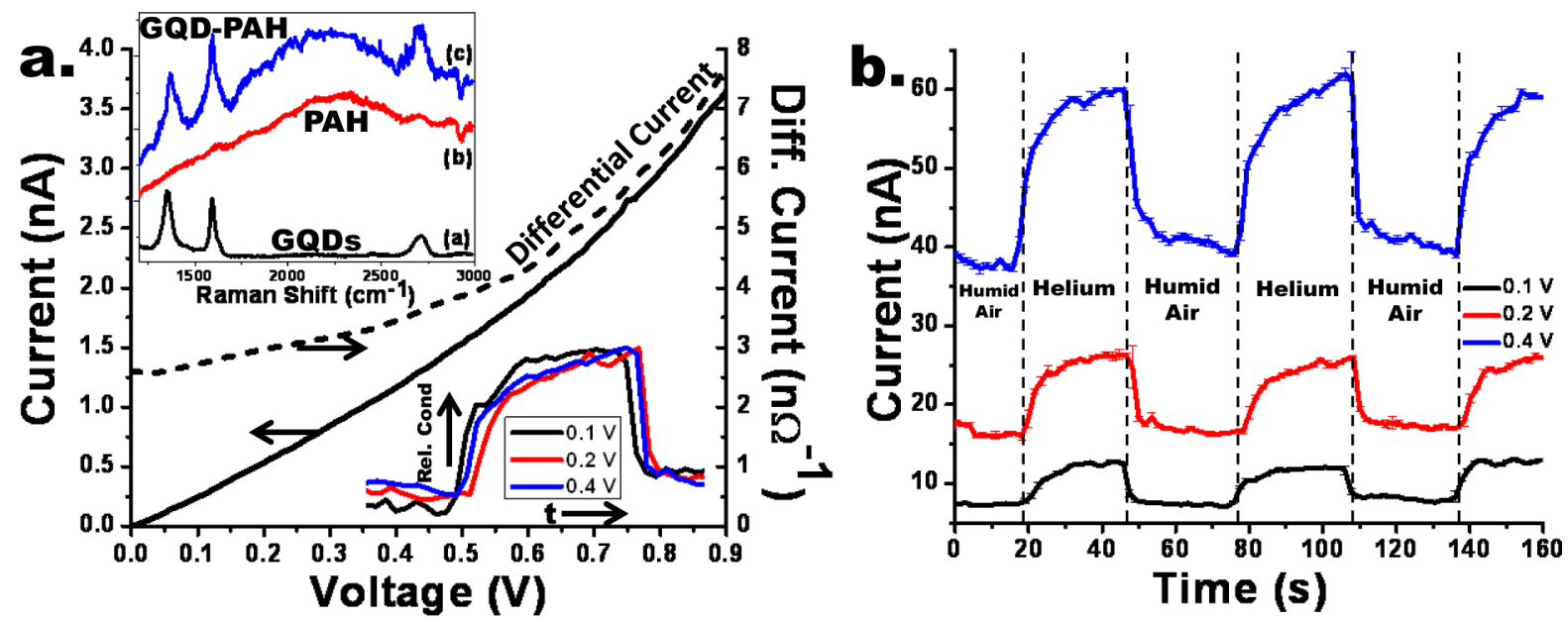

Figure 4. (a) Room-temperature current-voltage $(I-V)$ behavior of a PAH microfiber-GQD composite device, where the voltage increases from 0 to $0.9 \mathrm{~V}$ with a step size of $50 \mathrm{mV}$ ( $I-V$ at $80 \mathrm{~K}$ is presented in Supporting Information). The increase in the differential current (dI/dV) (dotted curve) depicts Coulomb blockade effect in the device. The top inset shows the Raman spectra of the PAH microfiber-GQD composite, parent PAH microfiber, and only GQDs, confirming GQD attachment on the microfiber. (b) Transient response of the currents of the PAH microfiber-GQD hybrid device with change in the local humidity. Multiple cycles of exposure of helium gas (30 s exposure time) at different bias voltages (0.1, 0.2, and $0.4 \mathrm{~V}$ ) points toward the robustness, sensitivity, and fast response of the device. The normalized transient currents with humidity at different voltages constructed from $b$ are shown in the bottom inset of part a. The consistency in the normalized transient conductivity implies a linear scaling of the response with voltage.

generating a strain resulting in the initiation of a cleavage-crack on the $\mathrm{C}-\mathrm{C}$ matrix. ${ }^{30}$ The propagation of this crack occurs via opening of the adjacent epoxy groups or the hopping of an epoxy group. Here, sequential oxidation of epoxy to diones is energetically favorable, especially in a dehydrating medium (conc: $\left.\mathrm{H}_{2} \mathrm{SO}_{4}\right){ }^{29}$ Further, the ketonic groups projecting outward on neighboring carbon atoms destabilizes the adjacent $\mathrm{C}-\mathrm{C}$ double bond, making it prone to oxidation, and subsequent cleavage. Similarly, ketones' conversion to carboxylic acids, and rearrangement leads to edge-roughening. ${ }^{31}$ Additionally, $\mathrm{KMnO}_{4}$ can form a manganate ester on graphene (similar to CNT oxidation), ${ }^{29}$ which upon oxidation forms dione and undergoes a similar cleavage pathway.

To incorporate GQDs into applications, their assembly into easy-to-fabricate devices is important. Here, we also report on a unique electron-tunneling modulation based device and sensor built via self-assembly of GQDs on hygroscopic polymer microfibers between electrodes. The device fabrication involves two steps: (a) spinning (Figure 3a,b) and immobilizing (Figure $3 \mathrm{c}, \mathrm{d})$ a polyallylmine hydrochloride $(\mathrm{PAH})$ microfiber on a silica substrate with predeposited gold electrodes $10 \mu \mathrm{m}$ apart, and (b) covalent anchoring of GQDs on the PAH microfibers (Figure 3e,f). Briefly, the silica substrate with predeposited gold electrodes was washed with deionized (DI) water, acetone, ethanol, and isopropanol (IPA) and dried using nitrogen gas flow. The chip was then immersed in a piranha solution at 50 ${ }^{\circ} \mathrm{C}$ inside a fume hood for $3 \mathrm{~h}$, followed by washing with water and IPA, and drying in nitrogen flow. The chip was further treated with oxygen plasma to make the surface of the chip slightly negatively charged (via addition of hydroxyl groups). A $40 \%$ solution of positively charged polyelectrolyte PAH solution was then spun to form microfibers (Figure 3a and b), which were placed across the electrodes on the negatively charged silica chip. The substrate was subsequently baked at $120^{\circ} \mathrm{C}$ for $6 \mathrm{~h}$ to bond the microfiber to the silica surface and to partially cross-link the PAH microfiber to avoid it being washed away during subsequent processing. Finally, the chip was washed with copious amounts of deionized (DI) water to remove excess unbound $\mathrm{PAH}$ and dried in nitrogen.

To covalently anchor GQDs on the PAH microfibers, an amine coupling agent 2-(7-aza-1H-benzotriazole-1-yl)-1,1,3,3tetramethyluronium hexafluorophosphate (HATU) was employed. Here, the chip with PAH microfibers (spun from $40 \%$ $\mathrm{PAH}$ solution in water) was submerged in a $20 \mathrm{~mL}$ of negatively charged GQD solution (prepared earlier) with $5 \mathrm{mg}$ of HATU added. The mixture was incubated at $30{ }^{\circ} \mathrm{C}$ for $12 \mathrm{~h}$ to produce strong and stable covalent bonds between the amine groups on PAH and the carboxylic acid groups on the GQDs. After the reaction, the chip was washed repeatedly with DI water. Figure $3 \mathrm{c}$ and $\mathrm{d}$ shows a picture and an optical micrograph of the fabricated device. The covalent bond is expected to form a very stable hybrid structure. Further, electrostatic repulsion between GQDs and the negatively charged silica ensures highly selective deposition of GQDs on $\mathrm{PAH}$. Figure $3 \mathrm{~g}$ shows a schematic diagram of the device construct. The electron micrographs of the GQD-PAH microfiber-composite confirmed the anchoring of GQDs onto the microfiber (Figure 3f). Further, the top inset of Figure 4a shows the Raman spectra of the microfiber, GQDs, and the composite of $\mathrm{GQD} /$ microfiber, where the $\mathrm{D}, \mathrm{G}$, and $2 \mathrm{D}$ peaks of the GQDs emerge on the fluorescent peak of the PAH microfiber, confirming the attachment of GQDs on the microfiber.

Electrical measurement on the GQD-microfiber device (shown later) suggests assembly of GQDs' percolating network linked via PAH molecular junctions (GQD-PAH-GQD junctions). Under the influence of an electric field, the electrons tunnel through the PAH barrier from one GQD to the next. These measurements were conducted by connecting the chip-electrodes with probes linked to a Keithley source meter to measure the tunneling current versus voltage and the transient response with respect to humidity and pressure (Figures $4 \mathrm{a}, \mathrm{b}$ and $5 \mathrm{a}$ ). All of the devices prepared by the above process were electrically annealed (applied a potential of $10 \mathrm{~V}$ for $10 \mathrm{~min}$ ) prior to the measurements. The electrical annealing 


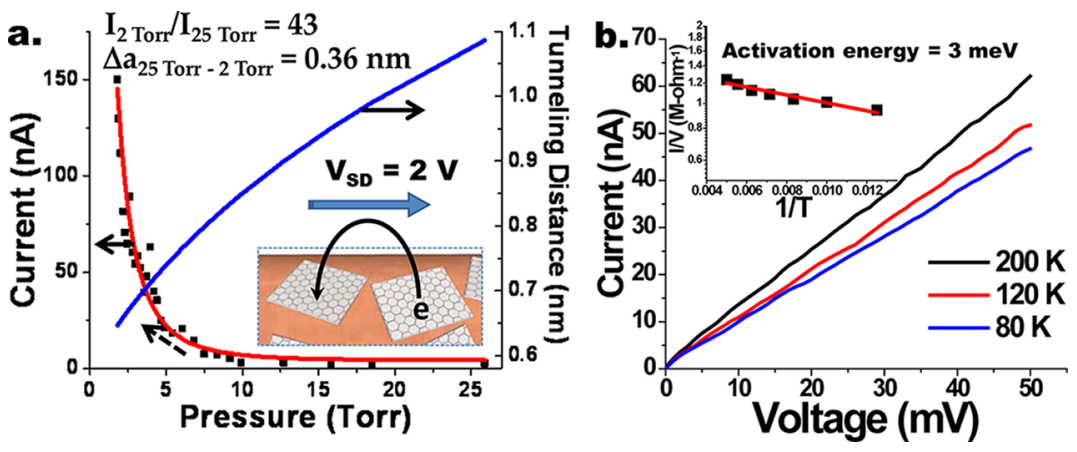

Figure 5. (a) The response of the electrical current in the percolating GQD-PAH-microfiber device at $2 \mathrm{~V}$ bias with the change in pressure (square points) is shown for a typical device. The model representing the combination of the Fowler-Nordheim electron tunneling and water transport via Henry's law fits the data with a regression of 0.9 (red curve). The model parameters obtained were used to plot the change in tunneling distances as a function of pressure (blue curve). (b) The influence of temperature on the electrical currents in the percolating-GQD device was studied. The current-voltage $(I-V)$ curves were obtained at 80,120 , and $200 \mathrm{~K}$, where the voltage increases from 0 to $50 \mathrm{mV}$. (Top, inset) The decrease in conductivity with decrease in temperature was also plotted with the Arrhenius fit (red curve) (data points = 80, 100, 120, 140, 160, 180, and 200 K). The data suggests an activation energy of $3 \mathrm{meV}$.

was important to allow the GQDs on the device to reach an equilibrium position. Once the equilibrium is reached, the device conductivity and response becomes robust. After annealing, the current-voltage $(I-V)$ characteristics of the device having PAH microfiber-GQD composite across the electrodes were generated using a two-point conductivity measurement and a voltage ramping from 0 to $0.9 \mathrm{~V}$ with a step size of $50 \mathrm{mV}$ (Figure 4a).

The IV exhibits a nonlinear behavior, more explicitly shown in the differential current $(\mathrm{d} I / \mathrm{d} V)$ curve. This is an indication of $2 \mathrm{D}$ Coulomb blockade effect, ${ }^{32-34}$ where the combined and nonsynchronous electrostatic charging/discharging occurs to transport the charges between neighboring GQDs with finite capacitances. The increase in currents reduces the electron residence time and thus the repulsive force on the upstream electrons, thereby increasing the rate of current-increase ( $\mathrm{d} I$ / $\mathrm{d} V$ ) with voltage. This process is modeled via the $2 \mathrm{D}$ Coulombblockade theory by Likharev: $I \propto\left(V / V_{T}-1\right)^{\zeta} .35$ To study this, we conducted an IV measurement with a four-point probe setup (to eliminate contact resistance) at $77 \mathrm{~K}$. The Likharev $\mathrm{fit}^{35}$ produced a blockade threshold voltage for our GQDdevice of $8 \mathrm{meV}$ (at $77 \mathrm{~K}(6.4 \mathrm{meV})$ ) and a geometry factor, $\zeta$, of 3 (Supporting Information, Figure S7). The geometry factor, $\zeta$, of 3 is consistent with reduced-graphene-oxide modeled as a GQD network, where $\zeta$ is $3.1-3.4^{34}$ Also, a quantized Coulomb staircase is not observed due to nonsynchronous electrostatic charging/discharging based transport through $2 \mathrm{D}$ GQD network with different sizes and capacitances $\left(\mathrm{C}_{\mathrm{GQD}}\right)$ with low charging energy (for example, $E_{\mathrm{C}}=\mathrm{e}^{2} / C_{\mathrm{GQD}}=3.5$ $\mathrm{meV}$ for $180 \mathrm{~nm} \mathrm{GQD}{ }^{36}$ ). The activation energy of our device was measured to be $3 \mathrm{meV}$ (shown later).

The total conductivity of the device is directly proportional to the probability of electron tunneling, which is exponentially and inversely proportional to the tunneling distance. Since the $\mathrm{PAH}$ microfiber is hygroscopic and at positive humid environment comprises of an additional volume of fused water, the change in relative humidity or water vapor pressure changes the volume of the water in the microfiber following the Henry's law, $V_{\mathrm{w}} / V_{\mathrm{f}}=H P$, where $V_{\mathrm{w}}$ and $V_{\mathrm{f}}$ are the volume of water and polymer in the microfiber, while $H$ and $P$ are the Henry's constant and water-vapor pressure, respectively).

To demonstrate the sensitivity of the device to humidity, we show the transient response of the current at a fixed voltage by changing the local humidity around the device. This was achieved by replacing humid air with He gas. Figure $4 \mathrm{~b}$ shows the increase in conductivity of the device during intervals of $\mathrm{He}$ exposure at three different voltages. The helium dehumidified chamber was maintained for $30 \mathrm{~s}$ intervals. The normalized response of the device (dividing the current with the average base current values) in Figure 4a, bottom inset, shows that the response is linearly scaled with voltage. The increase in current with the onset of helium exposure to the end of the interval indicates diffusion-limited process kinetics; while the helium concentration increases inside the chamber, the microfiber's water content still needs to desorb and diffuse out of the microfiber. Similar results were observed for exposure to argon and nitrogen as well.

Here, the change in the water volume (due to change in local water-vapor pressure) in the microfiber translates into a change in the tunneling distance between the GQDs: $a / a_{0}=(1+$ $H P)^{1 / 3}$, where $a$ and $a_{0}$ are the average tunneling distances at any point and at zero water-vapor pressure, respectively. The current values are governed by Fowler Nordhiem electron tunneling: ${ }^{37} I=I_{0}+T \exp \left(-\left(2(2 m \phi)^{1 / 2} / \hbar\right) a_{0}(1+H P)^{1 / 3}\right)$, where $T, m$, and $\phi$ are tunneling proportionality constants, mass of an electron, and tunneling barrier height.

To further quantify the system and to study the response of the device under direct influence of water-vapor pressure, another GQD-PAH device was connected to a vacuum probestation. Figure 5a shows the response of the device currents to the change in pressure for a typical GQD microfiber device and the associated data-fit curve (Henry's law and Fowler Nordhiem fit with regression $=0.9$ ). The vacuum was allowed to increase slowly, and the complete measurement was conducted over an hour to negate mass-transfer limitations. Based on the tunneling barrier of $5.11 \mathrm{eV}$ for the PAH polymeric junction, ${ }^{37}$ a tunneling distance of $0.58 \mathrm{~nm}$ for waterless microfiber was calculated (from data fit; Figure 5a). This value, along with the Henry's constant of 0.215 Torr $^{-1}$ (also from Figure 5a), suggests that from 25 to 2 Torr the average tunneling distance decreases by $0.36 \mathrm{~nm}$ (Figure 5a), while the conductivity increases by 43 -fold. Figure 5 a also shows the calculated change in the electron-tunneling distances with pressure, based on the model fit.

To eliminate the possibility of water-doping induced conductivity-modulation, we studied the response to pressure 
of a device with only GQDs and no PAH fiber (Supporting Information, Figure S8). From 25 to 2 Torr, the GQD-only device exhibited a negligible change in conductivity in comparison to the 43-fold increase shown by the GQD-PAH device. This indicates that the device response is not due to water adsorption and doping. Further, the graphene-dopingbased humidity-detection produces <6-fold change, in the reverse direction, and requires large humidity (60-90\% $\mathrm{rH}),{ }^{19,20}$ while in the current device the humidity range is $<40 \% \mathrm{rH} \quad(<0.007 \mathrm{~kg} / \mathrm{kg}$ with highest conductivity at lowest humidity). Further, only a PAH fiber device was also tested and exhibited a six-order-of-magnitude lower conductivity and a reverse response to humidity (i.e conductivity reduces at lower humidity). This is expected for ionic conductivity and further implies that the PAH is hygroscopic and must absorb/desorb water (leading to modulation of tunneling barrier width in our device). Therefore, ionic conductivity and its response is six orders of magnitude lower than conduction through the GQD network. These control experiments indicate that the primary cause of conductivity modulation with humidity in the GQD$\mathrm{PAH}$ device is the change in the tunneling-distance between GQDs. The experiments also show that the device functions very sensitively to humidity when both GQDs and PAH are present.

To ensure that the device undergoes electron-tunneling process, we studied the dependence of the device conductivity on (cryogenic) temperatures (at $1 \mathrm{mT}$ Torr vacuum). Figure $5 \mathrm{~b}$ shows the current-voltage $(I-V)$ characteristics of the device (GQD network on PAH microfiber) across the electrodes 10 $\mu \mathrm{m}$ apart with a voltage ramping from 0 to $50 \mathrm{mV}$ at 80,120 , and $200 \mathrm{~K}$. The inset shows the $(I / V)$ values plotted against $1 /$ $T$ to fit the Arrhenius plot: $\left(I / V \propto \exp \left(-E_{\mathrm{a}} / k_{\mathrm{B}} T\right)\right)$. The activation energy, $E_{a}$ is found to be $3 \mathrm{meV}$, which is significantly less than the $k_{\mathrm{B}} T$ at room temperature $(=25$ $\mathrm{meV}$ ), indicating that the mode of electron transport at room temperature is electron-tunneling, and not thermionic emission.

In summary, we demonstrate (a) the mechanism of edgeroughening and sequential oxidative-cleavage of GNRs into GQDs, (b) the characterization of their structural properties, (c) their selective assembly on a polyelectrolyte microfiber to produce percolating network exhibiting e-tunneling transport and Coulomb blockade, (d) functioning of GQD-based electronic humidity and pressure sensors, and (e) theoretical model explaining the relationship between water-mass-transfer from the polymer and electron-transport through the GQDs. The Raman $\left(I_{\mathrm{D}} / I_{\mathrm{G}}\right)$ and DLS (size distribution) show progressive reduction in GNR size with the propagation of the cleavage reaction. The GQDs deposited on hygroscopic polymer microfiber functions as an electrically percolating device, where electrons transport from one GQD to another via $\mathrm{PAH}$ molecular junctions. We show that $0.36 \mathrm{~nm}$ reduction in tunneling barrier width between GQDs increases the conductivity of the device by 43 -fold. We envision that a control on electron-tunneling through GQDs on smart, responsive polymer films or fibers will lead to development of a wide range of sensors, devices, and GQD system.

\section{ASSOCIATED CONTENT}

\section{(S Supporting Information}

Detailed methods, optical images of the fiber-GQD device, high-magnification TEM images of GQDs at different times of reaction, TEM images of GQDs after completion of reaction, large area TEM images of isolated GQDs, dynamic light scattering data on the GQDs, Raman based estimate of relative thermal conductivity, electron-tunneling equation fit parameters and methods, Coulomb blockade and four-probe $I V$ curve for GQD-PAH device, and control experiments with only GQDs (no PAH fiber) device. This material is available free of charge via the Internet at http://pubs.acs.org.

\section{AUTHOR INFORMATION}

\section{Corresponding Author}

*E-mail: vberry@ksu.edu.

\section{Notes}

The authors declare no competing financial interest.

\section{ACKNOWLEDGMENTS}

V.B. thanks the financial support from NSF (CMMI-1054877, CMMI-0939523, and CMMI-1030963), Office of Naval Research (grant-N000141110767), Terry C. Johnson Center for Basic Cancer Research, and KSU start-up. V.B. also thanks Phong Nguyen and Donovan Briggs for their support with the electrical characterization and device fabrication.

\section{REFERENCES}

(1) Mohanty, N.; Moore, D.; Xu, Z.; Sreeprasad, T. S.; Nagaraja, A.; Rodriguez, A. A.; Berry, V. Nanotomy-based production of transferable and dispersible graphene nanostructures of controlled shape and size. Nat. Commun. 2012, 3, 844.

(2) Novoselov, K. S.; Jiang, D.; Schedin, F.; Booth, T. J.; Khotkevich, V. V.; Morozov, S. V.; Geim, A. K. Two-dimensional atomic crystals. Proc. Natl. Acad. Sci. U.S.A. 2005, 102, 10451-10453.

(3) Bunch, J. S.; Yaish, Y.; Brink, M.; Bolotin, K.; McEuen, P. L. Coulomb oscillations and Hall effect in quasi-2D graphite quantum dots. Nano Lett. 2005, 5, 287-290.

(4) Libisch, F.; Stampfer, C.; Burgdorfer, J. Graphene quantum dots: Beyond a Dirac billiard. Phys. Rev. B 2009, 79, 115423.

(5) Ritter, K. A.; Lyding, J. W. The influence of edge structure on the electronic properties of graphene quantum dots and nanoribbons. Nat. Mater. 2009, 8, 235-242.

(6) Sreeprasad, T. S.; Berry, V. How Do the Electrical Properties of Graphene Change with its Functionalization? Small 2012, 9, 341-350.

(7) Lu, J.; Yeo, P. S. E.; Gan, C. K.; Wu, P.; Loh, K. P. Transforming C60 molecules into graphene quantum dots. Nat. Nanotechnol. 2011, 6, 247-252.

(8) Pan, D. Y.; Zhang, J. C.; Li, Z.; Wu, M. H. Hydrothermal Route for Cutting Graphene Sheets into Blue-Luminescent Graphene Quantum Dots. Adv. Mater. 2010, 22, 734.

(9) Andres, R. P.; Datta, S.; Dorogi, M.; Gomez, J.; Henderson, J. I.; Janes, D. B.; Kolagunta, V. R.; Kubiak, C. P.; Mahoney, W.; Osifchin, R. F.; Reifenberger, R.; Samanta, M. P.; Tian, W. Room temperature Coulomb blockade and Coulomb staircase from self-assembled nanostructures. J. Vac. Sci. Technol. A 1996, 14, 1178-1183.

(10) Beck, J. D.; Shang, L.; Marcus, M. S.; Hamers, R. J. Manipulation and real-time electrical detection of individual bacterial cells at electrode junctions: A model for assembly of nanoscale biosystems. Nano Lett. 2005, 5, 777-781.

(11) Berry, V.; Saraf, R. F. Self-assembly of nanoparticles on live bacterium: An avenue to fabricate electronic devices. Angew. Chem., Int. Ed. 2005, 44, 6668-6673.

(12) Berry, V.; Rangaswamy, S.; Saraf, R. F. Highly selective, electrically conductive monolayer of nanoparticles on live bacteria. Nano Lett. 2004, 4, 939-942.

(13) Berry, V.; Gole, A.; Kundu, S.; Murphy, C. J.; Saraf, R. F. Deposition of CTAB-terminated nanorods on bacteria to form highly conducting hybrid systems. J. Am. Chem. Soc. 2005, 127, 1760017601 . 
(14) Peet, J.; Heeger, A. J.; Bazan, G. C. "Plastic" Solar Cells: SelfAssembly of Bulk Heterojunction Nanomaterials by Spontaneous Phase Separation. Acc. Chem. Res. 2009, 42, 1700-1708.

(15) Maheshwari, V.; Saraf, R. F. High-resolution thin-film device to sense texture by touch. Science 2006, 312, 1501-1504.

(16) Liu, G.; Rumyantsev, S.; Shur, M.; Balandin, A. A. Graphene thickness-graded transistors with reduced electronic noise. Appl. Phys. Lett. 2012, 100, 033103.

(17) Rumyantsev, S. L.; Liu, G.; Shur, M. S.; Balandin, A. A. Observation of the memory steps in graphene at elevated temperatures. Appl. Phys. Lett. 2011, 98, 222107-3.

(18) Rumyantsev, S.; Liu, G.; Shur, M. S.; Potyrailo, R. A.; Balandin, A. A. Selective Gas Sensing with a Single Pristine Graphene Transistor. Nano Lett. 2012, 12, 2294-2298.

(19) Massera, E.; La Ferrara, V.; Miglietta, M.; Polichetti, T.; Nasti, I.; Di Francia, G. Gas sensors based on graphene. Chem. Today 2011, 29, 39-41.

(20) Ghosh, A.; Late, D. J.; Panchakarla, L. S.; Govindaraj, A.; Rao, C. N. R. $\mathrm{NO}_{2}$ and humidity sensing characteristics of few-layer graphenes. J. Exp. Nanosci. 2009, 4, 313-322.

(21) Hummers, W. S.; Offeman, R. E. Preparation of Graphitic Oxide. J. Am. Chem. Soc. 1958, 80, 1339.

(22) Mohanty, N.; Nagaraja, A.; Armesto, J.; Berry, V. HighThroughput, Ultrafast Synthesis of Solution-Dispersed Graphene via a Facile Hydride Chemistry. Small 2009, 6, 226-231.

(23) Kudin, K. N.; Ozbas, B.; Schniepp, H. C.; Prud'homme, R. K.; Aksay, I. A.; Car, R. Raman spectra of graphite oxide and functionalized graphene sheets. Nano Lett. 2008, 8, 36-41.

(24) Cai, W.; Moore, A. L.; Zhu, Y.; Li, X.; Chen, S.; Shi, L.; Ruoff, R. S. Thermal Transport in Suspended and Supported Monolayer Graphene Grown by Chemical Vapor Deposition. Nano Lett. 2010, $10,1645-1651$.

(25) Lu, J.; Yeo, P. S. E.; Gan, C. K.; Wu, P.; Loh, K. P. Transforming C60 molecules into graphene quantum dots. Nat. Nanotechnol. 2011, 6, 247-252.

(26) Pan, D.; Zhang, J.; Li, Z.; Wu, M. Hydrothermal Route for Cutting Graphene Sheets into Blue-Luminescent Graphene Quantum Dots. Adv. Mater. 2010, 22, 734-738.

(27) Li, Y.; Hu, Y.; Zhao, Y.; Shi, G.; Deng, L.; Hou, Y.; Qu, L. An Electrochemical Avenue to Green-Luminescent Graphene Quantum Dots as Potential Electron-Acceptors for Photovoltaics. Adv. Mater. 2011, 23, 776-780.

(28) Peng, J.; Gao, W.; Gupta, B. K.; Liu, Z.; Romero-Aburto, R.; Ge, L.; Song, L.; Alemany, L. B.; Zhan, X.; Gao, G.; Vithayathil, S. A.; Kaipparettu, B. A.; Marti, A. A.; Hayashi, T.; Zhu, J. J.; Ajayan, P. M. Graphene Quantum Dots Derived from Carbon Fibers. Nano Lett. 2012, 12, 844-849.

(29) Kosynkin, D. V.; Higginbotham, A. L.; Sinitskii, A.; Lomeda, J. R.; Dimiev, A.; Price, B. K.; Tour, J. M. Longitudinal unzipping of carbon nanotubes to form graphene nanoribbons. Nature 2009, 458, 872-876.

(30) Li, J. L.; Kudin, K. N.; McAllister, M. J.; PrudÆhomme, R. K.; Aksay, I. A.; Car, R. Oxygen-driven unzipping of graphitic materials. Phys. Rev. Lett. 2006, 96, 176101.

(31) Wolfe, S.; Ingold, C. F.; Lemieux, R. U. Oxidation of olefins by potassium permanganate. Mechanism of .alpha.-ketol formation. J. Am. Chem. Soc. 1981, 103, 938-939.

(32) Stampfer, C.; Guttinger, J.; Molitor, F.; Graf, D.; Ihn, T.; Ensslin, K. Tunable Coulomb blockade in nanostructured graphene. Appl. Phys. Lett. 2008, 92, 012102.

(33) Jasuja, K.; Thompson, A.; Berry, V. Reversibly Compressible and Stretchable 'Spring-like' Polymeric Nano-Junctions between Metal Nanoparticles. Small 2008, 4, 2181-2186.

(34) Joung, D.; Zhai, L.; Khondaker, S. I. Coulomb blockade and hopping conduction in graphene quantum dots array. Phys. Rev. B 2011, 83, 115323.

(35) Rimberg, A. J.; Ho, T. R.; Clarke, J. Scaling Behavior in the Current-Voltage Characteristic of One- and Two-Dimensional Arrays of Small Metallic Islands. Phys. Rev. Lett. 1995, 74, 4714-4717.
(36) Guttinger, J.; Stampfer, C.; Hellmuller, S.; Molitor, F.; Ihn, T.; Ensslin, K. Charge detection in graphene quantum dots. Appl. Phys. Lett. 2008, 93, 212102.

(37) Berry, V.; Saraf, R. F. Modulation of Electron Tunneling in a Nanoparticle Array by Sound Waves: An Avenue to High-Speed, High-Sensitivity Sensors. Small 2011, 7, 2485-2490. 